\title{
Etude de la structure des formations végétales à Afzelia africana Smith et Isoberlinia doka Craib \& Stapf dans le parc national du $\mathbf{W}$ du Niger.
}

\author{
Douka Mahaman Sanoussi ${ }^{1}$, Dahiratou Ibrahim Doka ${ }^{1}$, Moussa Barage ${ }^{2}$ \\ 1 Laboratoire de mycologie Ecole Normale Supérieure Université Abdou Moumouni de Niamey Niger \\ 2 Faculté d'agronomie Université Abdou Moumouni de Niamey Niger \\ Correspondance: sanoussidouka@yahoo.fr \\ Mots-clés : Isoberlinia doka, Afzelia africana, parc du W du Niger. \\ Keywords: Isoberlinia doka, Afzelia africana, Niger Park W.
}

Publication date 31/08/2019, http://www.m.elewa.org/JAPS

\section{RESUME}

Cette étude porte sur deux espèces ligneuses à savoir Afzelia. africana et Isoberlinia. doka qui sont classées sur la liste des espèces vulnérables et menacées dans le monde. Elle a permis d'estimer l'état actuel desdites espèces dans le parc national de $W$. Ces espèces sont menacées de disparition dans toute l'Afrique. L'objectif visé a été d'étudier la structure de leur population dans le Parc National du W du Niger. Pour ce faire, trente (30) placettes carrées de dimension $30 \mathrm{~m} * 30 \mathrm{~m}$ ont été installées suivant les pistes tracées du Parc W que nous avons considérées comme des transects. Les relevés phytosociologiques ont été réalisés selon la méthode sigmatiste de Braun-Blanquet (1932). L'installation de la placette dépend de la présence de l'une des deux espèces où de toutes les deux. Les placettes sont distantes de $500 \mathrm{~m}$ les une des autres. La superficie totale inventoriée était de 2,7 ha. Dans les placettes toutes les espèces ont été recensées et les mesures dendrométriques ont porté sur $A$. africana et d'I. doka. Au total 51 espèces reparties en 41 genres et 21 familles ont été recensées. En termes d'abondance, les familles les mieux représentées sont les Combretaceae (17,31\%), les Mimosaceae (15,38\%), les Ceasalpinoidea $(9,61 \%)$. Dans la famille des cesalpinoidea les espèces Afzelia africana et Isoberlinia doka ont un taux de représentions de 5,64\% dont Afzelia africana avec un taux de représentation de 4,07\% contre 1,57\% pour Isoberlinia doka. L'indice de diversité spécifique de Shannon et l'équitabilité de Pielou sont respectivement de 1,38 bit et de 0,8 ; ce qui montre que la végétation est moyennement diversifiée. La densité d' $A$. africana est de 45,17 pieds/ha et 17,49 pieds/ha chez $I$. doka, la régénération est de l'ordre de 75,92 pieds/ha chez $A$. africana et 31,11 pieds/ha chez I. doka. Les dimensions en diamètre et en hauteur pour chacune des espèces sont illustrées par des histogrammes en forme de cloche à travers l'ajustement de Weibull avec des dissymétries gauches caractéristiques des individus relativement jeunes. L'ajustement de Weibull montre également que les distributions en diamètre des deux espèces sont identiques. Les modes de régénération par drageons sont plus observés chez les deux espèces avec peu de cas de germination par graines chez A. africana. Cette étude a permis d'avoir des connaissances sur l'état actuel sur la présence desdites espèces au parc W. Cette étude a permis de disposer des connaissances scientifiques sur les deux populations et donc de proposer des mesures idoines de gestion durable des ces espèces dans les réserves périphériques au Niger. 


\section{ABSTRACT}

This study focuses on two woody species namely Afzelia. africana and Isoberlinia. doka which are on the list of vulnerable and endangered species worldwide. It has made it possible to estimate the current status of these species in the National Park W. These species are threatened with extinction throughout Africa. The aim was to study the structure of their population in Niger's National Park W. To do this, thirty (30) square plots of $30 \mathrm{~m} *$ $30 \mathrm{~m}$ size were installed along the marked tracks of Park $W$ that we considered as transects. The phytosociological surveys were carried out according to the sigmatist method of BraunBlanquet (1932). The installation of the plot depends on the presence of one of the two species or both. The plots are $500 \mathrm{~m}$ apart from each other. The total area surveyed was 2.7 ha. In the plots all species were recorded and the dendrometric measurements were carried out on $A$. africana and I. Doka. A total of 51 species divided into 41 types and 21 families were identified. In terms of abundance, the best represented families are Combretaceae $(17.31 \%)$, Mimosaceae $(15.38 \%)$, and Ceasalpinoidea $(9.61 \%)$. In the family of the Cesalpinoidea species Afzelia africana and Isoberlinia doka have a representation rate of $5.64 \%$ including Afzelia africana with a representation rate of $4.07 \%$ against $1.57 \%$ for Isoberlinia doka. The Shannon specific diversity index and the Pielou equitability are respectively 1.38 bit and 0.8 ; which shows that the vegetation is moderately diversified. The density of $A$. africana is 45.17 feet / ha and 17.49 plants / ha in I. doka, regeneration is in the order of 75.92 plants / ha in A. africana and 31.11 plants / ha in I. doka .The diameter and height dimensions for each of the species are illustrated by bell-shaped histograms through Weibull adjustment with left asymmetries characteristic of relatively young individuals. The Weibull adjustment also shows that the diameter distributions of the two species are identical. The sucker regeneration modes are more observed in both species with few cases of seed germination in $\boldsymbol{A}$. africana. This study made it possible to have knowledge of the current state of the presence of these species at Park W. This study made it possible to obtain scientific knowledge on the two populations and thus to propose appropriate measures of these species sustainable management in the peripheral reserves in Niger.

\section{INTRODUCTION}

Les forêts tropicales sont les plus diversifiées, mais aussi les plus menacées. Le plus important facteur de l'appauvrissement actuel de la diversité biologique de ces écosystèmes est leur fragmentation. Selon FAO (2010), la perte annuelle du couvert forestier tropical a été évaluée à 13 millions d'hectares entre 20002010 et l'Afrique ayant seulement $16,8 \%$ des forêts mondiales a contribué pour $27 \%$ à cette réduction. Les forêts d'Afrique les plus diversifiées, du moins les plus riches en espèces ligneuses, sont celles des régions centrales (la forêt du Mont Cameroun, le Sud-est du Gabon), et du Nord-est de Madagascar (Natta, 2003). En Afrique de l'Ouest, la discontinuité connue sous le nom du couloir sec «DahomeyGap », interrompt les forêts denses humides tropicales entre le Sud-est du Ghana et le Sudouest du Nigeria. Les pays sahéliens d'Afrique de l'Ouest, tel que le Niger en sont exclus et ne disposent pour ainsi d'aucune forêt dense humide. Compte tenu de sa position dans la zone tropicale sèche, le Niger n'échappe pas à la profonde dégradation de ces écosystèmes. $\mathrm{La}$ FAO (2010), estime la perte annuelle en superficie des forêts naturelles à 37050 ha. La situation actuelle, d'autant plus préoccupante avec la crise alimentaire et les incertitudes climatiques, contraignent les populations rurales dont la majorité se trouve sous le seuil de pauvreté, à exercer une plus grande pression sur les ressources naturelles. Le Parc National du W du Niger situé à la lisière du Sahel contient des exemples représentatifs de types de zones 
humides des savanes d'Afrique Occidentale. Il offre un atout exceptionnel de conservation et d'utilisation durable des zones humides en milieu semi-aride. La zone du Parc W est une zone transfrontalière partagée entre le Bénin, le Burkina et le Niger. Elle renferme une végétation riche et variée (RAMSAR, 2004). Plus de 500 espèces de plantes ont été décrites à ce jour. Six (6) espèces parmi les plus courantes et facilement reconnaissables sont Adansonia digitata, Balanites aegyptiaca, Piliostigma reticulatum, Borassus aethiopum Bombax costatum et Parkia biglobosa. Les pressions anthropiques et autres facteurs de diverses natures agissant sur la structure des formations végétales étant constants dans le temps sans que d'importantes actions spécifiques de conservation ne soient

\section{MATERIELS ET METHODE}

\subsection{Matériels}

3.1.1 Site d'étude : Le Parc National du W du Niger se situe dans la partie Sud-Ouest du pays entre les latitudes $11^{\circ}$ et $12^{\circ} 35$ Nord et les longitudes $2^{\circ}$ et $3^{\circ} 50$ Est. Il est situé à $150 \mathrm{~km}$ de Niamey la capitale avec une superficie de près de 220000 ha. Il est limité au Nord par la Tapoa, à l'est par le fleuve Niger, au Sud par la Mékrou (Bénin) et à l'ouest par le Parc d'Arly (Burkina Faso). Depuis sa création en 1954, cette aire protégée a acquis graduellement divers statuts : Zone Humide d'Importance Internationale, particulièrement pour les oiseaux d'eau en 1982 ; site Ramsar en 1987 ; Patrimoine Mondial de l'UNESCO en 1996. Il utilisées, l'Union Mondiale pour la Conservation de la Nature (IUCN) a dû classer en 1994, A. africana et $I$. doka sur la liste rouge des espèces vulnérables à protéger (Hawtorne, 1995). Une meilleure conservation de ces espèces dans un écosystème donné doit passer nécessairement par la connaissance de l'état actuel de leurs populations à travers l'étude structurale et l'état de régénération. Cette étude menée par la méthode sigmatiste de BraunBlanquet (1932) a permis d'une part d'évaluer l'état actuel desdites espèces dans le parc W dont Afzelia africana représente 4,07\% contre $1,57 \%$ pour Isoberlinia doka et d'autre part de montrer que les espèces $A$. africana et $I$. doka se régénèrent surtout par drageonnage mais avec peu de germination par graines pour $A$. africana.

appartient à la catégorie II de l'UICN (2002). Cette requalification juridique a permis au PNWN de bénéficier d'une protection intégrale où toute exploitation extractive est proscrite. Sa protection a été renforcée ensuite par la création d'autres Réserves à la périphérie permettant une réduction de la pression humaine. Il est ainsi encadré par la Réserve Totale de Faune de Tamou (77 000 ha) et la Réserve Partielle de Dosso (306 000 ha). Malgré les difficultés inhérentes à la préservation des espaces protégés en Afrique, le PNWN semble avoir joué son rôle restrictif vis-à-vis des exploitations agricoles et forestières. 


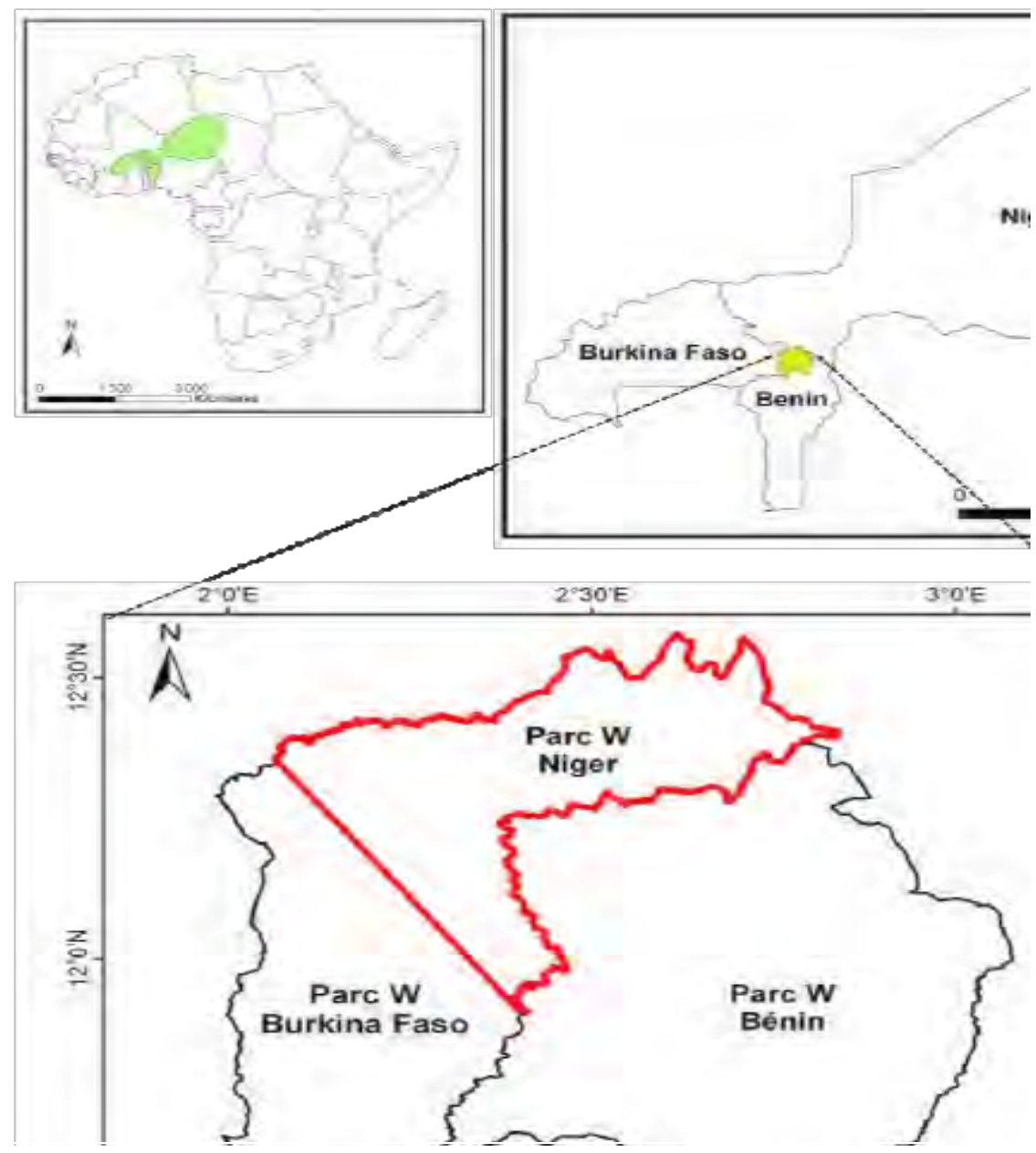

Figure 1 : Localisation géographique du parc National du W du Niger (PNWN). (CSIG ECOPAS)

Le climat est continental de type soudanosahélien. Il est marqué par une courte saison des pluies, allant de juin à septembre, et une longue saison sèche de novembre à avril. Le gradient pluviométrique s'étage de sa partie septentrionale avec une pluviométrie moyenne annuelle cumulée de $685 \mathrm{~mm}$ à $850 \mathrm{~mm}$ dans sa partie la plus méridionale (Benoît, 1998). L'évapotranspiration potentielle (ETP), au niveau de la station météorologique de la Tapoa (1981-2002), est maximale de mars (274 mm) à mai $(270 \mathrm{~mm})$, et atteint sa plus faible valeur en août (206 mm). Couteron et al., (1992) et Boudouresque (1995) ont fait un bilan de la documentation cartographique disponible pour la région d'étude. Les principaux types de sols sont :
- les sols minéraux bruts situés sur matériaux durs ;

- les sols peu évolués d'érosion lithique sur relief accidenté où la roche affleure à maints endroits ;

- les sols peu évolués d'apport (alluvionscolluvions) qui présentent toujours des traces d'hydromorphie temporaire ;

- les sols ferrugineux tropicaux ;

- les sols ferralitiques.

Dans le parc W la composition et la structure de la végétation diffèrent selon un gradient nord-sud. La végétation est une mosaïque de forêts et de savanes à Combretaceae (Couteron et al., 1992 ; Mahamane, 2005). Cette hétérogénéité du paysage est très liée au relief et au type de sol (Arbonnier, 2002 ; Mahamane 2005). Dans cette mosaïque on rencontre 
également, les végétations périodiques sur les plateaux à sol peu profond (Barbier, 2006 ; Diouf, 2010). La flore du Parc National du W du Niger comporte plus de 1000 espèces de phanérogames endémiques ou semiendémiques (White, 1983). Le Parc National du W est parcouru par 4 cours d'eau principaux : le fleuve Niger et 3 de ses affluents (Tapoa, Mékron et Alibor). Le fleuve Niger comporte deux régimes, la crue en décembre janvier, déterminée par les pluies qui surviennent dans son bassin supérieur en Guinée, et l'étiage en mars, avril, mai et juin. La saison des pluies est caractérisée par une petite crue ou crue locale déterminée par les pluies qui tombent sur son bassin dans la zone. Quant aux affluents, ce sont des cours d'eau temporaires qui connaissent leur crue en saison des pluies

3.2. Matériel de terrain: Le matériel de terrain et de collecte des données est constitué des outils ci-dessous énumérés :

- Un GPS pour la prise des coordonnées géographiques ;

- Des rubans-mètre de $50 \mathrm{~m}$ et $1,5 \mathrm{~m}$ ont été utilisés respectivement pour la délimitation des placettes et pour la mesure des circonférences des deux espèces à 1,30 $\mathrm{m}$ de hauteur;

- Des piquets pour matérialiser les côtés des placettes ;

- Des jalons pour la mesure des hauteurs totales des arbres d'A. africana et d'I. doka;

- Une machette pour se frayer des layons et débroussailler le pourtour des arbres à mesurer ;
- Des fiches d'inventaire des ligneux ;

- Un appareil photo numérique pour la prise de photos des formations.

\subsection{Description des deux espèces}

3.3.1 Afzelia africana: Le genre Afzelia appartient à l'ordre des Fabales, famille des Leguminoseae (Fabaceae), sous-famille des Ceasalpinioideae. A. africana est un grand arbre de 25 à $30 \mathrm{~m}$ voire $35 \mathrm{~m}$ de haut, à cime dense, large et étalée ; le fût est droit à diamètre moyen variant entre $60 \mathrm{~cm}$ et $90 \mathrm{~cm}$ mais pouvant atteindre les $2 \mathrm{~m}$. Ses contreforts ont une hauteur comprise entre $1 \mathrm{~m}$ et 1,5 m. C'est un arbre à branches massives et à écorce grise plus ou moins foncée, relativement fissurée et lamelleuse, s'écaillant en plaques, laissant apparaître des zones gris-claires, granuleuses à tranche rose à brun-claire granuleuse. Cette dernière caractéristique participe à sa pyrorésistance (Ahouangonou et Bris, 1997). Son feuillage est vert brillant; les feuilles alternes, paripennées mesurant jusqu'à $30 \mathrm{~cm}$ de long, portent 3 à 8 paires de folioles opposées ou subopposées, glabres et luisantes, vert-foncé au-dessus, largement espacées sur le rachis. L'inflorescence est une panicule terminale d'environ $20 \mathrm{~cm}$ de long ; les fleurs sont blanches, striées de pourpre, à trois pétales supérieurs elliptiques de 10 à $12 \mathrm{~mm}$ de long, très odorantes et un pétale inférieur à 2 lobes. Les fruits sont des gousses aplaties ligneuses, glabres, de 10 à $18 \mathrm{~cm}$ x 6 à $8 \mathrm{~cm}$, épaisses de 2 à $5 \mathrm{~cm}$, à deux valves noires, contenant 7 à 10 graines noires, lisses plus ou moins brillantes. 


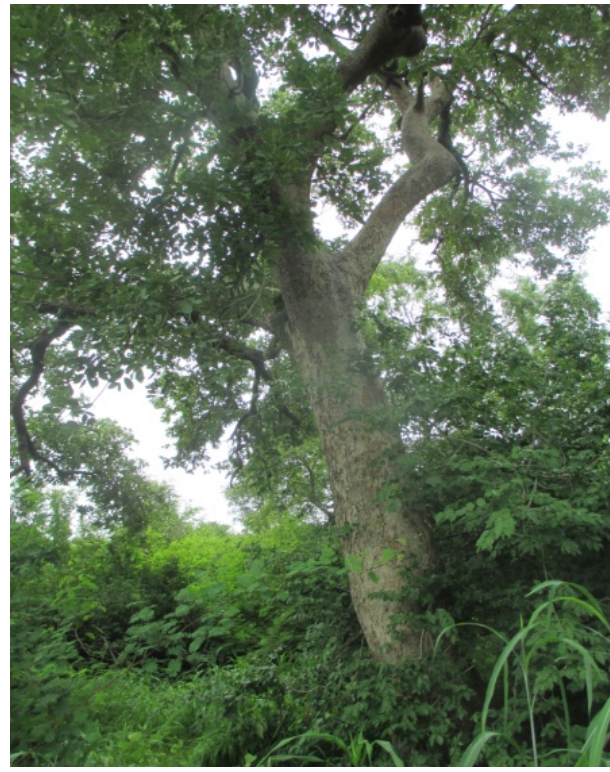

A.

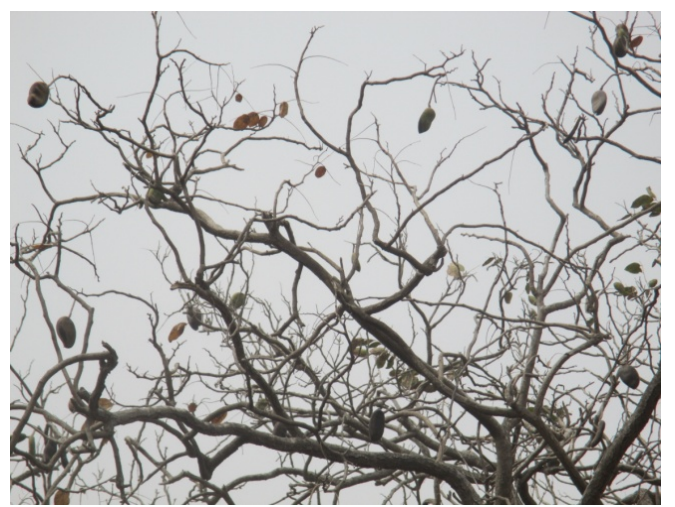

C.

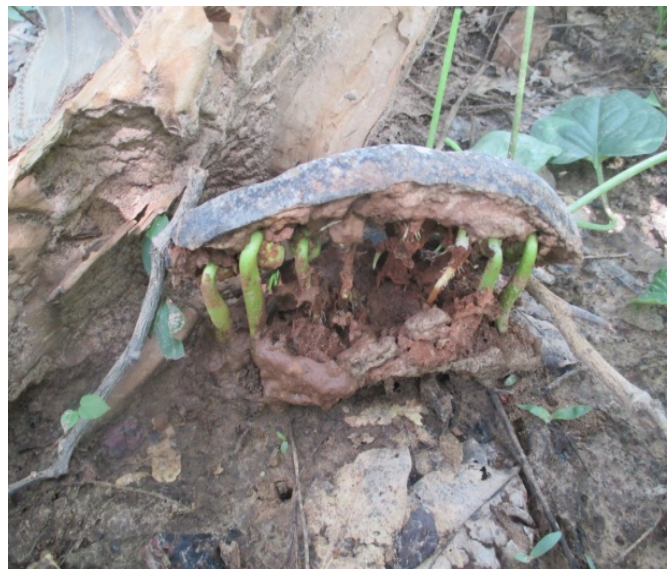

E.

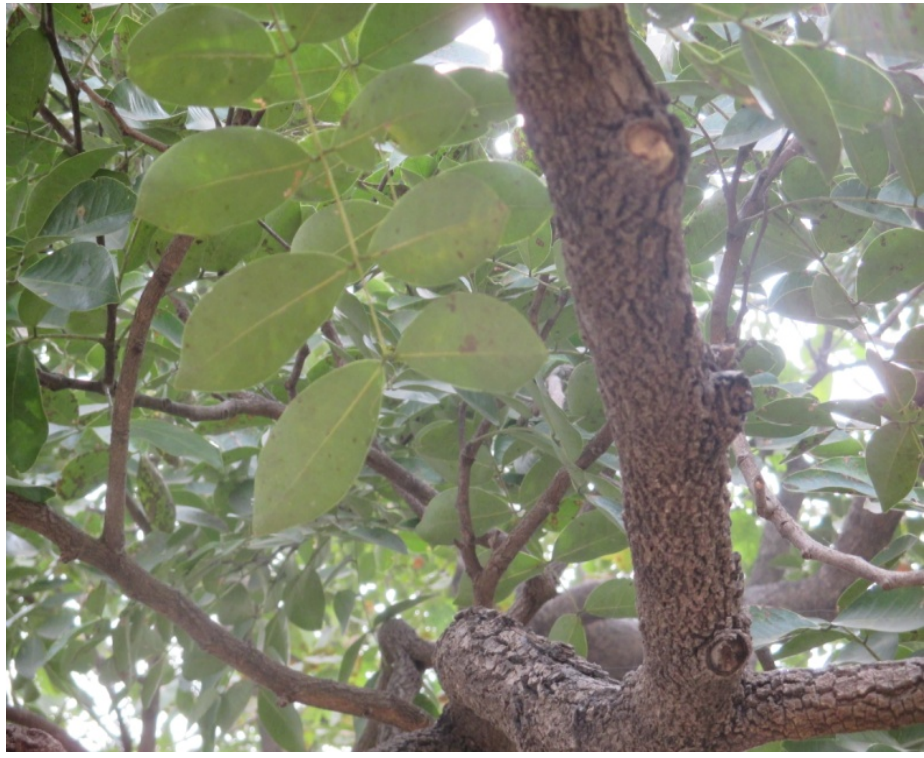

B.

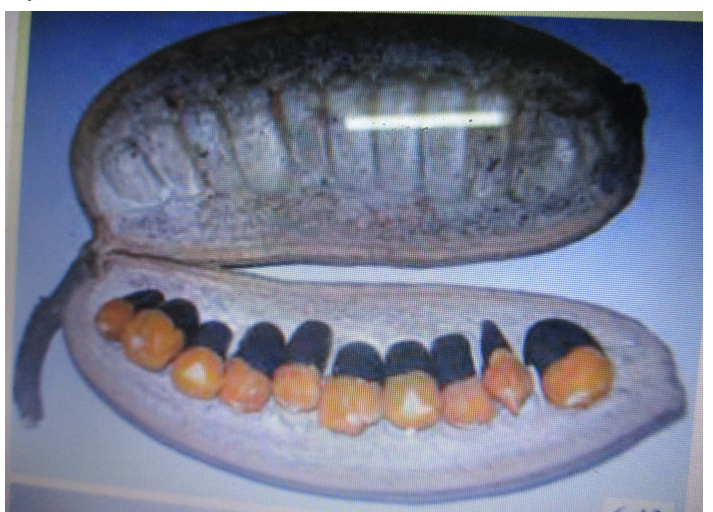

D.

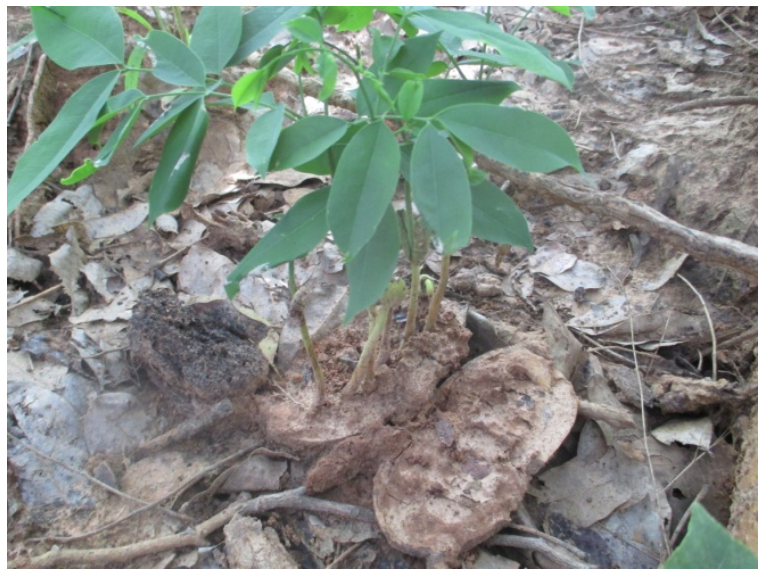

F.

Légende :(source : Douka Mahaman Sanoussi): Pied d'Afželia africana (A), forme d'écorce d'Afzelia africa (B), Afzelia africana en fructification (C), Graines d'Afzelia africana (D), Graines d'A. africana en Germination car ayant bénéficier de la Protection de la coque sous un peuplement de semenciers (E\&F). 


\subsubsection{Isoberlinia doka: Le genre Isoberlinia} appartient à l'ordre des Fabales, famille des Leguminoseae (Fabaceae), sous-famille des Ceasalpinioideae. $I$. doka est un arbre de 10 à 15 $\mathrm{m}$ de haut, pouvant mesurer parfois $20 \mathrm{~m}$, avec un diamètre atteignant 60 à $80 \mathrm{~cm}$ à l'âge adulte. La cime de $I$. doka est étroite et ouverte, l'écorce du tronc est grise, écailleuse, à tranche rouge clair (Arbonnier, 2009). L'appareil végétatif comprend deux à cinq feuilles jugulées à stipules inter foliaires falciformes et caduques.
Les feuilles sont alternes, paripennées, de 15 à $30 \mathrm{~cm}$ de long, aux folioles opposées, ovales ou elliptiques, parfois oblongues. Les inflorescences sont groupées en panicules. Les fleurs blanches subsessiles sont enveloppées de bractéoles valvaires constituées de cinq sépales linéaires subégaux, de cinq pétales égaux et de 10 à 13 étamines exsertes libres et subégales. Les fruits sont des gousses plates ligneuses de 15 à $30 \mathrm{~cm}$ sur 4 à $7 \mathrm{~cm}$ s'ouvrant en spirales bivalves (Geerling, 1982).

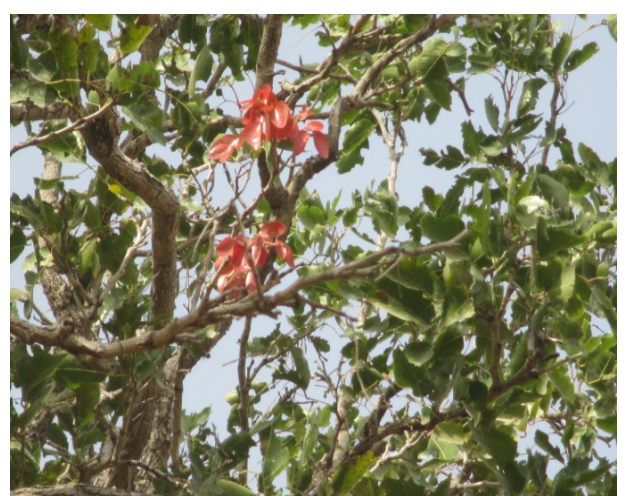

A

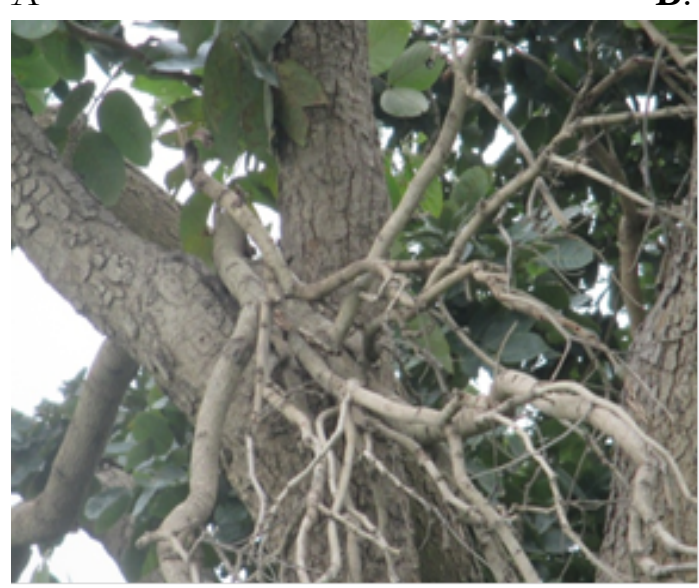

D.

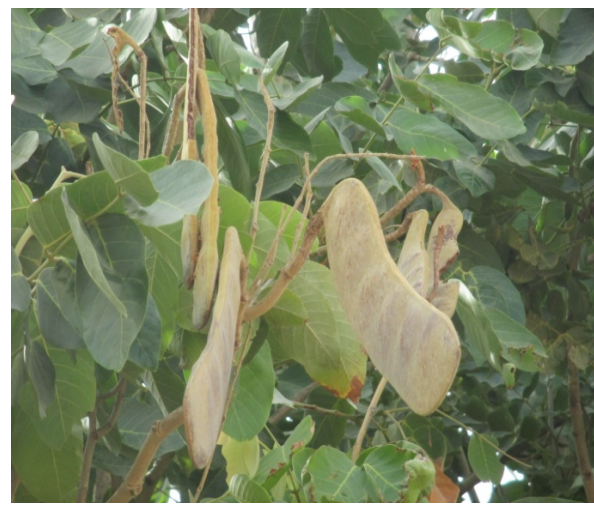

B.

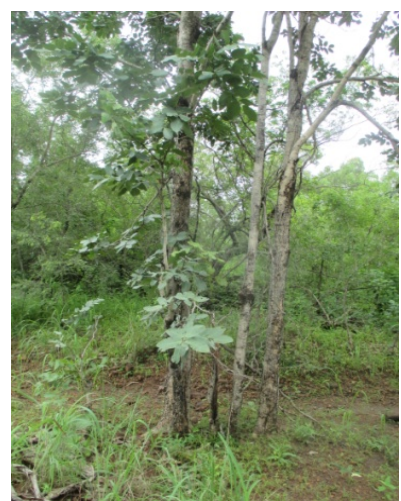

C

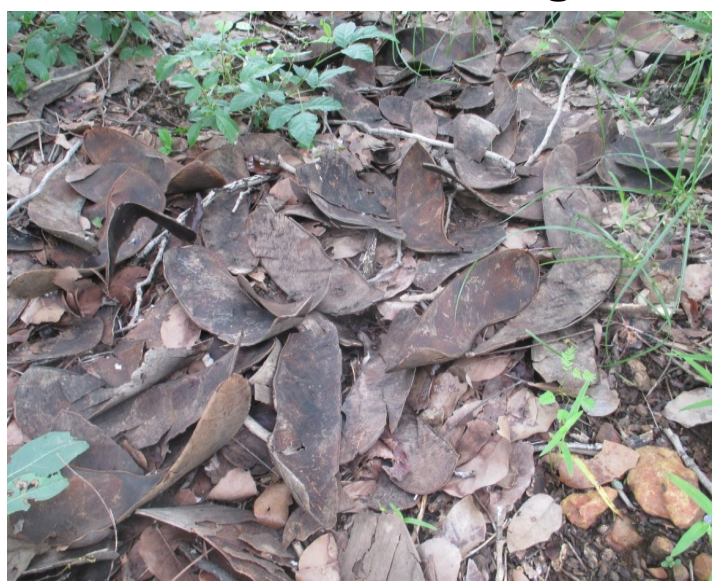

E.

Légende :(source : Douka Mahaman Sanoussi): Pied d'Isoberlinia doka (A), Isoberlinia doka en fructification (B), I. doka régénère par drageonnage sous un peuplement de semenciers (C), forme d'écorce Isoberlinia doka (D), coques d'I. doka sous un peuplement de semenciers sans régénération par graines (E).

\subsubsection{Méthode: Les relevés} phytosociologiques ont été réalisés selon la méthode sigmatiste de Braun-Blanquet (1932) sur la base de l'homogénéité floristique et écologique des stations (Gounot, 1969). Cette méthode permet de décrire les communautés végétales et de comprendre l'organisation spatio-temporelle sur le plan quantitatif et qualitatif des espèces constituantes (Houinato, 2001). Ainsi le relevé a été réalisé à travers un échantillonnage orienté dans des placettes de $900 \mathrm{~m} 2(30 \mathrm{~m}$ x $30 \mathrm{~m})$. L'installation de la 
placette dépend de la présence de l'une des deux espèces où de toutes les deux. Nous avons considéré les pistes tracées du parc W comme les transects lors de l'installation des placettes. Les placettes sont distantes de $500 \mathrm{~m}$ les une des autres. Dans chaque placette, tous les arbres sont recensés. Les pieds d'Isoberlinia doka ou d'Afrelia africana sont mesurés à $1,30 \mathrm{~m}$, les ramifications en plusieurs branches en dessous de 1,30m sont considérées comme des rejets et si elles dépassent $1,30 \mathrm{~m}$, les pieds qui les comportent sont dits des multicaules. Dans chaque placette de relevés, des mesures dendrométriques classiques ont été portées sur les pieds d'Isoberlinia doka ou d'Afželia africana. Les autres ligneux se trouvant dans la placette sont tout simplement recencées. Les paramètres suivants ont été mensurés : la hauteur, les deux diamètres perpendiculaires du houppier, le diamètre des arbres à hauteur d'homme (1,30 $\mathrm{m})$. Pour les pieds multicaules, seul le plus gros tronc a été mensuré. Les traces d'émondage et d'écorchage sont notées. Pour le cas spécifique du parc W les placettes sont distancées de $300 \mathrm{~m}$ les unes des autres, les pieds d'Isoberlinia doka et d'Afzelia africana qui sont entre les placettes sont simplement recensés.

\section{$4 \quad$ Analyse des données}

4.1 Composition floristique : La composition floristique a été déterminée pour l'ensemble du peuplement arborescent des forêts claires inventoriées à travers les paramètres suivants :

4.1.1 Richesse floristique : Nombre total des espèces recensées.

Abondance des espèces : nombre d'individus d'une espèce /nombre total des individus recensés x100.

4.1.2 Densité des espèces : nombre d'individus d'une espèce sur la superficie totale inventoriée.

4.2 Diversité spécifique et équitabilité : L'analyse de la diversité spécifique a été faite par le calcul de l'indice de diversité de ShannonWeaver et l'indice de Simpson, d'équitabilité de Pielou et la richesse spécifique.

L'indice de Shannon (H):
4.2.1 $\mathbf{H}=-\Sigma$ pi $\log 2$ pi. Avec pi $=$ ni $/ \mathrm{N}$, abondance relative de l'espèce i dans l'échantillon total auquel appartient l'espèce, $\mathrm{N}=$ effectif total des individus, $\mathrm{ni}=$ effectif des individus de l'espèce $i$. L'indice $\mathrm{H}$ est exprimé en bit et est utilisé pour apprécier l'hétérogénéité et la diversité d'un biotope. $\mathrm{H}<$ $2,5=$ faible $; 2,5 \leq \mathrm{H}<4=$ moyen $; \mathrm{H} \geq 4=$ élevé

L'indice de Simpson (D) :

4.2.2 $\mathrm{D}=\Sigma$ pi (ni-1)/ $(\mathrm{N}-1)$. Cet indice a une valeur égale à 0 pour le maximum de dominance et une valeur de 1 pour le minimum de dominance. Dans le but d'obtenir des valeurs de diversité spécifique, on peut préférer l'indice de diversité d'après Simpson représenté par D-1; le maximum de diversité étant représenté par la valeur 1 et le minimum de diversité par la valeur 0 . Le mode de répartition des espèces au sein du groupement a été calculé à l'aide de l'équitabilité de Pielou (1966) qui est donnée par la formule suivante:

4.2.3 $\mathbf{E}=\mathbf{H} / \log 2 \mathrm{~S} . \quad \mathbf{E}=$ équitabilité de Pielou; $\mathbf{S}=$ nombre total d'espèces constitutives du groupement, $\log 2 \mathrm{~S}=$ diversité spécifique maximale de Shannon. L'indice $(\mathbf{E})$ traduit le degré de diversité atteint par rapport au maximum possible (Djégo (2006) in Morou, 2010) et varie de 0 à 1 . Il tend vers 0 lorsque la quasi-totalité des individus appartiennent à une seule espèce et prend la valeur 1 quand toutes les espèces ont les mêmes proportions.

$\mathrm{E}<0,6=$ faible $; 0,6 \leq \mathrm{E} \leq 0,7=$ moyen $; \mathrm{E} \geq$ $0,8=$ élevé.

4.3 Analyse de la structure de la végétation : L'étude du regroupement des tiges par catégories de grosseur présente un grand intérêt en matière de gestion forestière car elle est une expression de la structure du peuplement (Rondeux, 1999). L'ensemble des individus d'A. africana et d'I. doka, ont été répartis par classes de diamètre d'amplitude 6 $\mathrm{cm}$. Ainsi, la densité (en arbres/ha) par classes de diamètre a été déterminée. En ce qui concerne, la structure en hauteur, les arbres sont regroupés par catégories de hauteur. Les classes ainsi constituées sont d'amplitude $2 \mathrm{~m}$. 
Aussi, les fréquences absolues par classes de hauteur ont été déterminées et ont servi au calcul de la densité (en arbres/ha) par classes de hauteur. Les différents histogrammes construits ont été ajustés à la distribution de Weibull à 3 paramètres $(a, b$ et $c)$. Cette distribution assez couramment citée dans la littérature forestière se caractérise par une grande souplesse d'emploi. Sa fonction de densité de probabilité se présente sous la forme ci-dessous (Rondeux, 1999):

$$
(\boldsymbol{x})=(\boldsymbol{x}-\boldsymbol{a b})-1 \boldsymbol{e x p}[-(\boldsymbol{x}-\boldsymbol{a b}) \boldsymbol{c}]
$$

$x=$ diamètre (circonférence) des arbres;

$a=$ paramètre d'origine (ou de position); il est égal à 0 si toutes les catégories d'arbres sont considérées (des plantules jusqu'aux semenciers); il est non nul si les arbres considérés ont un diamètre supérieur ou égal à $a$; dans la présente étude, $a$ est égal à $6 \mathrm{~cm}$ dans le cas des structures en diamètre et égal à $2 \mathrm{~m}$ dans le cas des structures en hauteur des arbres. $b=$ paramètre d'échelle ou de taille; il est lié à la valeur centrale des diamètres ou circonférences des arbres du peuplement considéré.

$c=$ paramètre de forme lié à la structure en diamètre ou hauteur considérée. Pour chaque peuplement, les données de diamètre des arbres ont servi à l'estimation des paramètres $a, b$ et $c$ grâce à la méthode du maximum de vraisemblance disponible dans le langage MatLab (version R2006a). Des intervalles de confiance de ces estimations ont été aussi calculés. La distribution de Weibull peut prendre plusieurs formes selon la valeur du paramètre de forme $(c)$ :

\section{RESULTATS}

Au total, 51 espèces ont été recensées, reparties en 41 genres et 21 familles. Le nombre d'espèce varie selon la famille. Les familles les mieux représentées sont les Combretaceae (17,31\%), les Mimosaceae $(15,38 \%)$, les Ceasalpinoidea $(9,61 \%)$, les Rubiaceae $(5,77 \%)$, les Anacardiaceae (5,77\%) et les Fabaceae (5,77\%). Les quinze autres familles représentent 49,39\%. La densité moyenne a été évaluée à 400 individus par hectare dont 15,08\% pour $c \leq 1$ : la distribution est en « J renversée ", caractéristique des peuplements multispécifiques ou inéquiennes.

$c=1$ : la distribution est exponentiellement décroissante, caractéristique des populations en extinction.

$1 \leq c \leq 3,6$ : la distribution est asymétrique négative ou asymétrique gauche, caractéristique des peuplements monospécifiques avec prédominance d'individus jeunes ou de petits diamètres.

$c=3,6$ : la distribution est symétrique et la structure est normale, caractéristique des peuplements équiennes ou monospécifiques.

$c \geq 3,6$ : la distribution est asymétrique positive ou asymétrique droite, caractéristique des peuplements monospécifiques à prédominance d'individus âgés ou de gros diamètres. L'analyse log-linéaire (ou analyse de variance basée sur le maximum de variance) a été réalisée pour comparer dans chaque cas, la distribution en diamètre observée à la distribution théorique de Weibull avec estimation des paramètres. Cette méthode statistique est plus robuste que le traditionnel test d'ajustement de Pearson surtout en cas de faible effectif des classes (Agresti, 1990 ; Caswell 2001). L'état de régénération a été déterminé à travers un comptage systématique dans chaque placette des individus à $\mathrm{dbh}<6 \mathrm{~cm}$ des deux espèces. Ainsi, sont considérés comme faisant partis de la régénération, les arbres de diamètre $5 \mathrm{~cm}$ dans la zone soudanienne sèche et sahel (SUN, 2008).

Combretum nigricans, $14,07 \%$ pour Combretum molle, 6,59\% pour Feretia apodanthera, 6,03\% pour Boscia senegalensis et 5,57\% pour l'espèce Combretum collinum soit au total 47,34\% du peuplement. Tandis que $A$. africana et $I$. doka ne représentent respectivement que 4,07 et $1,57 \%$ avec des densités de 45,17 pied/ha pour $A$. africana et 17,49 pied/ha pour I. doka. Le milieu est diversifié avec un indice de Shannon $(\mathrm{H})$ de 1,38 bits $(\mathrm{H}<2,5)$. Cette dernière a été vérifiée 
en calculant l'indice de Simpson (D) dont sa réciproque $1-\mathrm{D}$ est égalé à 0,93 . Ces résultats permettent de conclure que le milieu présente une bonne diversité spécifique. Quant à l'équitabilité de Pielou $(\mathrm{H})$ elle est globalement élevée de l'ordre de $0,81(\mathrm{H}>0,8)$.

Tableau 1 : Répartition en familles et genres des espèces.

\begin{tabular}{|c|c|c|c|c|c|}
\hline Familles & Genre & Espèces & $\begin{array}{l}\text { EFFECTIF } \\
\mathrm{S}\end{array}$ & $\begin{array}{l}\text { ABONDANCE } \\
\text { RELATIVE (\%) }\end{array}$ & $\begin{array}{l}\text { DENSITE } \\
\text { PIED/HA }\end{array}$ \\
\hline Mimosoïdea & Acacia & Acacia ataxacanta & 2 & 0,07 & 0,73 \\
\hline Mimosoïdea & Acacia & Acacia erythrocalyx & 49 & 1,61 & 17,85 \\
\hline Mimosoïdea & Acacia & Acacia sieberiana & 5 & 0,16 & 1,82 \\
\hline Mimosoïdea & Acacia & Acacia macrostachia & 36 & 1,18 & 13,11 \\
\hline Ceasalpinioideae & Afzelia & Afzelia africana & 124 & 4,07 & 45,17 \\
\hline Annonaceae & Anogeisus & Anogeisus leocarpa & 102 & 3,34 & 37,16 \\
\hline Combretaceae & Anona & Anona senegalensis & 1 & 0,03 & 0,36 \\
\hline Balanitaceae & Balanites & Balanites aegyptiaca & 6 & 0,20 & 2,19 \\
\hline Bombaceae & Bombax & Bombax costatum & 33 & 1,08 & 12,02 \\
\hline Balanitaceae & Boscia & Boscia angustifolia & 61 & 2,00 & 22,22 \\
\hline Capparidaceae & Boscia & Boscia senegalensis & 184 & 6,03 & 67,03 \\
\hline Mimosoïdea & Cassia & Cassia seberiana & 15 & 0,49 & 5,46 \\
\hline Combretaceae & Combretum & Combretum aculatum & 15 & 0,49 & 5,46 \\
\hline Combretaceae & Combretum & Combretum collinum & 170 & 5,57 & 61,93 \\
\hline Combretaceae & Combretum & Combretum glutinosum & 128 & 4,20 & 46,63 \\
\hline Combretaceae & Combretum & Combretum micrantum & 51 & 1,67 & 18,58 \\
\hline Combretaceae & Combretum & Combretum mollé & 429 & 14,07 & 156,28 \\
\hline Combretaceae & Combretum & Combretum nigricans & 460 & 15,08 & 167,58 \\
\hline Rubiaceae & Crossopteryx & Crossopteryx febrifuga & 83 & 2,72 & 30,24 \\
\hline Caesalpiniaceae & Detarium & Detarium microcarpum & 102 & 3,34 & 37,16 \\
\hline Mimosaceae & dichrostachys & Dichrostachys cinerea & 108 & 3,54 & 39,34 \\
\hline Ebenaceae & Diospyros & Diospyros mespiliformis & 59 & 1,93 & 21,49 \\
\hline Rubiaceae & Feretia & Feretia apodanthera & 201 & 6,59 & 73,22 \\
\hline Euphobrbiaceae & Flueggea & Flueggea virosa & 61 & 2,00 & 22,22 \\
\hline Rubiaceae & Gardenia & Gardenia erubisceus & 14 & 0,46 & 5,10 \\
\hline Tiliaceae & Gardenia & Gardenia ternifolia & 23 & 0,75 & 8,38 \\
\hline Tiliaceae & Grewia & Grewia bicolor & 10 & 0,33 & 3,64 \\
\hline Combretaceae & Guiera & Guiera senegalensis & 34 & 1,11 & 12,39 \\
\hline Ceasalpinoidea & Isoberlinia & Isoberlinia doka & 48 & 1,57 & 17,49 \\
\hline Meliaceae & Khaya & Khaya senegalensis & 2 & 0,07 & 0,73 \\
\hline Bignoniaceae & Kigelia & Kigelia africana & 7 & 0,23 & 2,55 \\
\hline Anacardiaceae & Lannea & Lannea acida & 31 & 1,02 & 11,29 \\
\hline Anacardiaceae & Lannea & Lannea microcarpa & 2 & 0,07 & 0,73 \\
\hline Capparidaceae & Maerua & Maerua angolensis & 5 & 0,16 & 1,82 \\
\hline Celastraceae & Maytenus & Maytenus senegalensis & 1 & 0,03 & 0,36 \\
\hline Fabaceae & Ostryoderris & Ostryoderris stublmanii & 5 & 0,16 & 1,82 \\
\hline Mimosaceae & Parkia & Parkia biglobosa & 1 & 0,03 & 0,36 \\
\hline Ceasalpinoidea & piliostigma & Piliostigma reticulatum & 14 & 0,46 & 5,10 \\
\hline Mimosaceae & Prosopis & Prosopis africana & 29 & 0,95 & 10,56 \\
\hline Fabaceae & pterocarpus & Pterocarpus erinaceus & 3 & 0,10 & 1,09 \\
\hline Anacardiaceae & sclerocarya & Sclerocarya birrea & 3 & 0,10 & 1,09 \\
\hline Polygalaceae & securidaca & Securidaca longipedonculata & 35 & 1,15 & 12,75 \\
\hline Euphorbiaceae & securinega & Securinega virosa & 64 & 2,10 & 23,32 \\
\hline Sterculiaceae & Seterculia & Seterculia setigera & 5 & 0,16 & 1,82 \\
\hline Bignoniaceae & Stereospermum & Stereospermum kunthianum & 59 & 1,93 & 21,49 \\
\hline Loganiaceae & Strychnos & Strychnos spinosa & 12 & 0,39 & 4,37 \\
\hline Ceasalpinoidea & Tamarindis & Tamarindis indica & 17 & 0,56 & 6,19 \\
\hline Combretaceae & Terminalia & Terminalia avicennioides & 67 & 2,20 & 24,41 \\
\hline Sapotaceae & Vietellaria & Vitellaria paradoxa & 21 & 0,69 & 7,65 \\
\hline Fabaceae & Xeroderris & Xeroderris stublmanii & 28 & 0,92 & 10,20 \\
\hline Olacaceae & Ximenia & Ximenia americana & 12 & 0,39 & 4,37 \\
\hline Rhamnaceae & Ziziphus & Ziziphus micronata & 13 & 0,43 & 4,74 \\
\hline 21 & 41 & 51 & 050 & 0,00 & 11,11 \\
\hline
\end{tabular}


5.1 Analyse structurale des deux structures en diamètre et en hauteur des espèces : L'analyse structurale a porté sur les individus d'A. africana et d'I. doka.

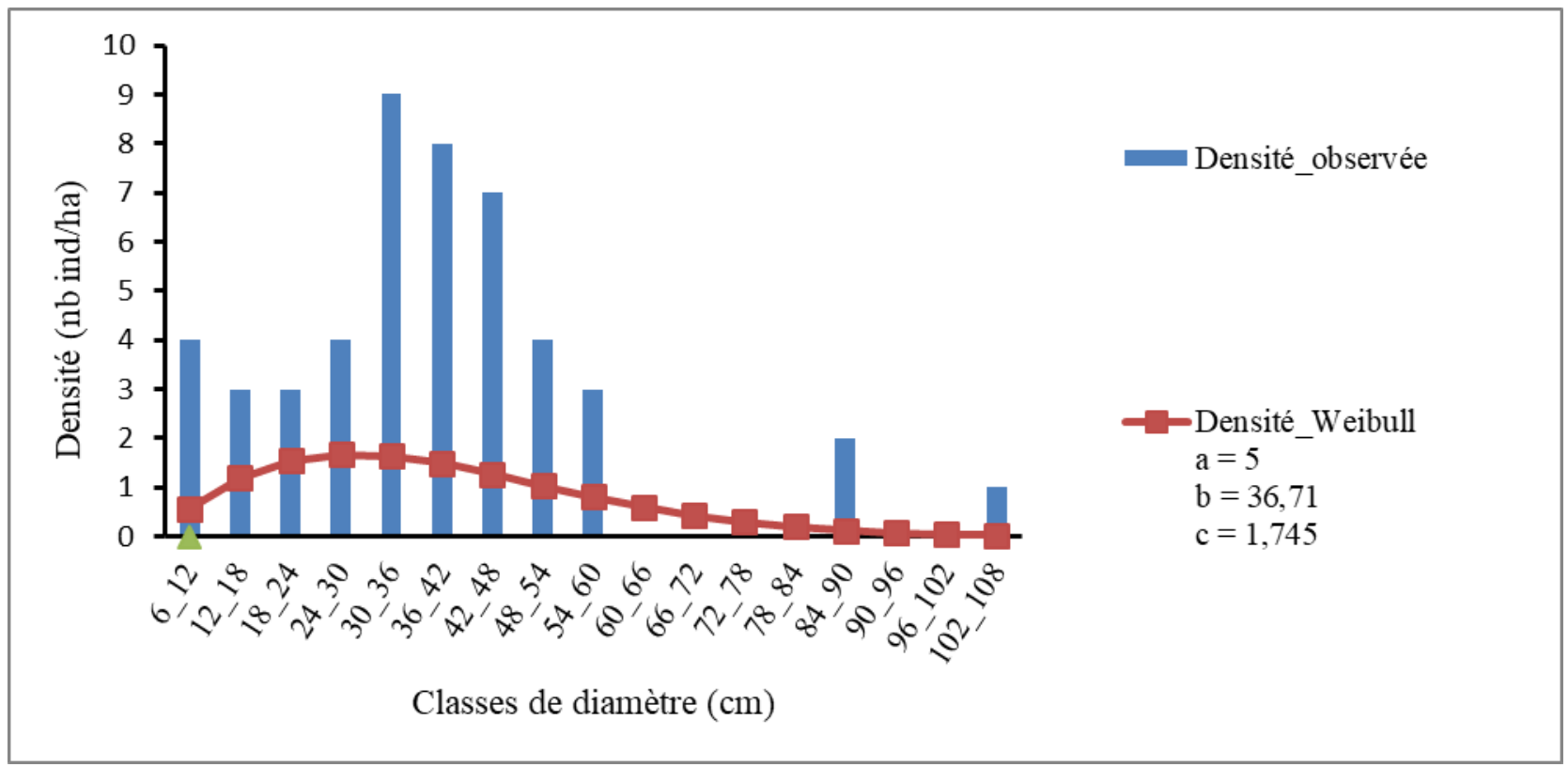

Figure 2 : Structure en diamètre de la population d'Afželia africana

L'analyse de la figure $\mathrm{n}^{\circ} 2$ selon weibull montre que les populations d'A. africana les plus représentées à l'hectare appartiennent respectivement aux classes de diamètre compris entre $24-30 \mathrm{~cm}$ et $30-36 \mathrm{~cm} ; 18-24 \mathrm{~cm}$ et $36-42$ $\mathrm{cm} ; 42-48 \mathrm{~cm} ; 48-54 \mathrm{~cm} ; 54-60 \mathrm{~cm}$. les individus de diamètre supérieur à $60 \mathrm{~cm}$ et inférieur à $12 \mathrm{~cm}$ sont quasi absents

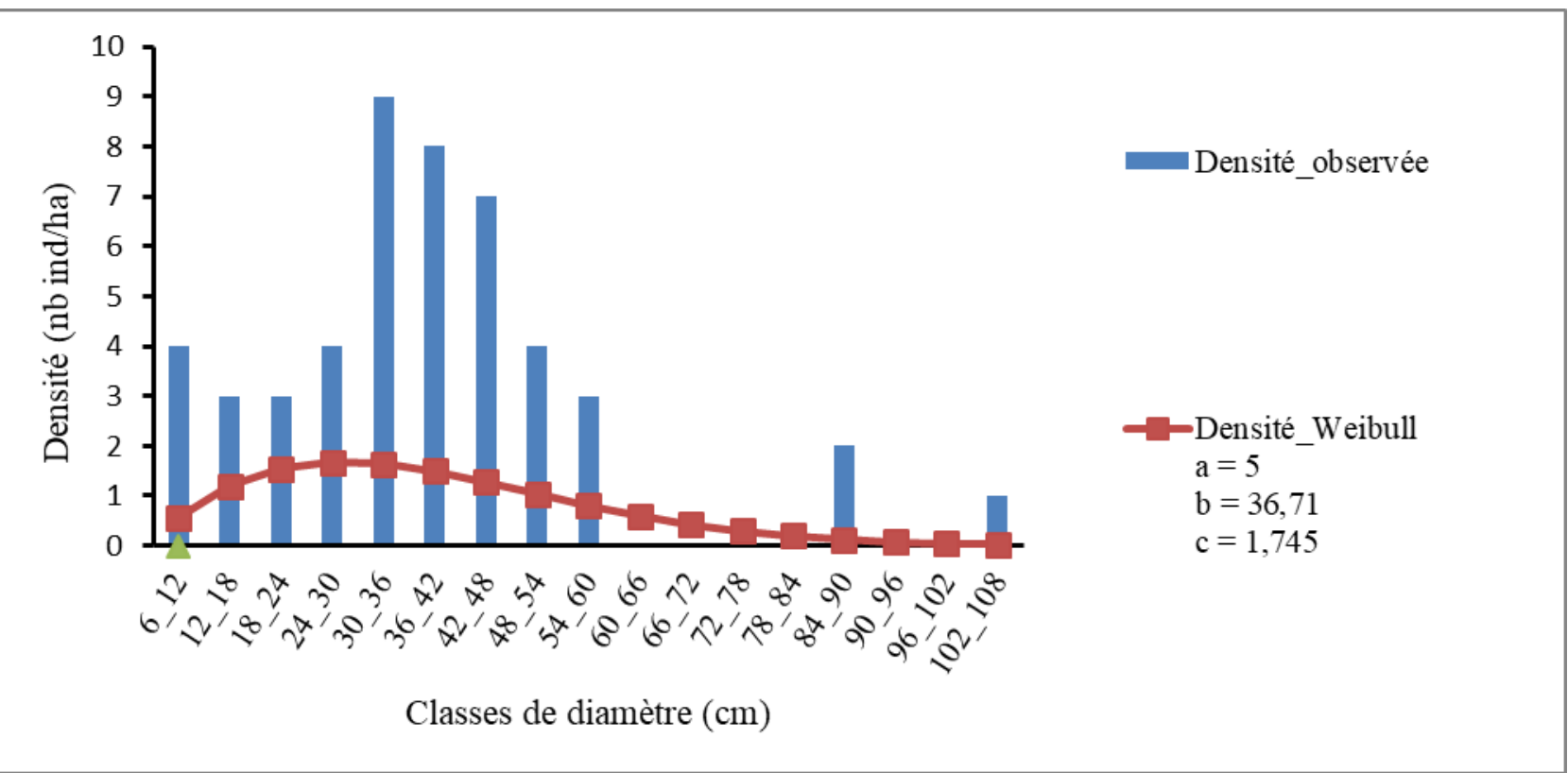

Figure 3 : structure en diamètre de la population d'Isoberlinia doka 
L'analyse de la figure $n^{\circ} 3$ selon weibull montre que les populations d'I. doka, les plus représentées à l'hectare appartiennent respectivement aux classes de diamètre compris entre $30-36 \mathrm{~cm}, 24-30 \mathrm{~cm}, 18-24 \mathrm{~cm}$ et $36-42$ $\mathrm{cm} ; 12-18 \mathrm{~cm}$ et $42-48 \mathrm{~cm} ; 48-54 \mathrm{~cm} ; 54-60$ $\mathrm{cm}$. Les individus de diamètre supérieur à 60 $\mathrm{cm}$ et inférieur à $12 \mathrm{~cm}$ de diamètre sont quasi absents.

5.2 Structures en hauteur: Les figures 4 et 5 , présentent les structures en hauteur des populations d' $A$. africana et I. doka.

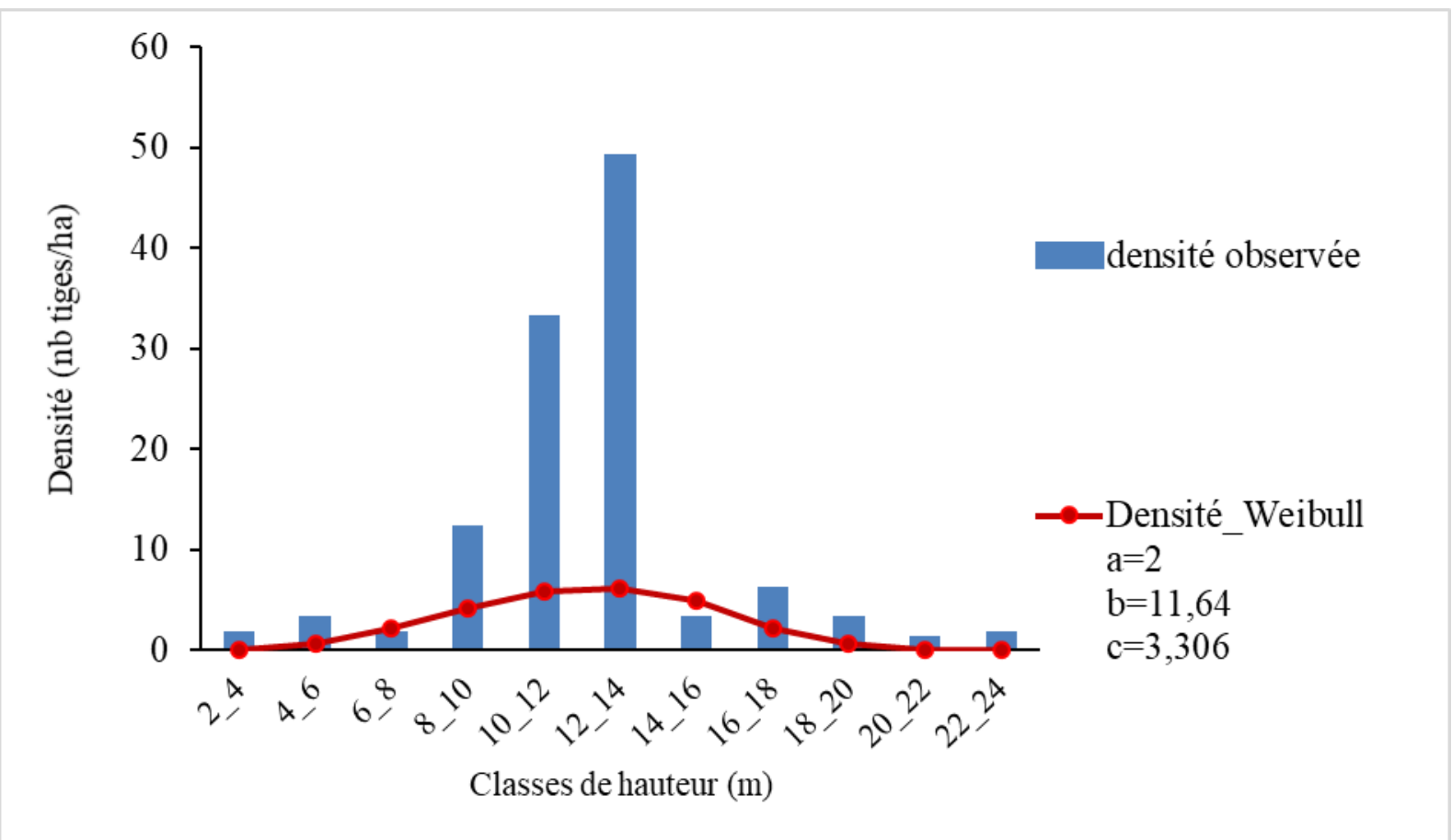

Figure 4 : Classes de hauteur d'Afželia africana

L'analyse de la figure $\mathrm{n}^{\circ} 4$ montre que les individus de la population d' $A$. africana les plus représentés à l'hectare appartiennent respectivement aux classes de hauteur comprise entre $10-12 \mathrm{~m}$ et $12-14 \mathrm{~m}, 8-10 \mathrm{~m}, 14-16 \mathrm{~m}, 6-8$ $\mathrm{m}$ et 16-18 $\mathrm{m}$. Les individus de taille supérieure à $18 \mathrm{~m}$ et inférieure à $6 \mathrm{~m}$ sont quasi absents. 


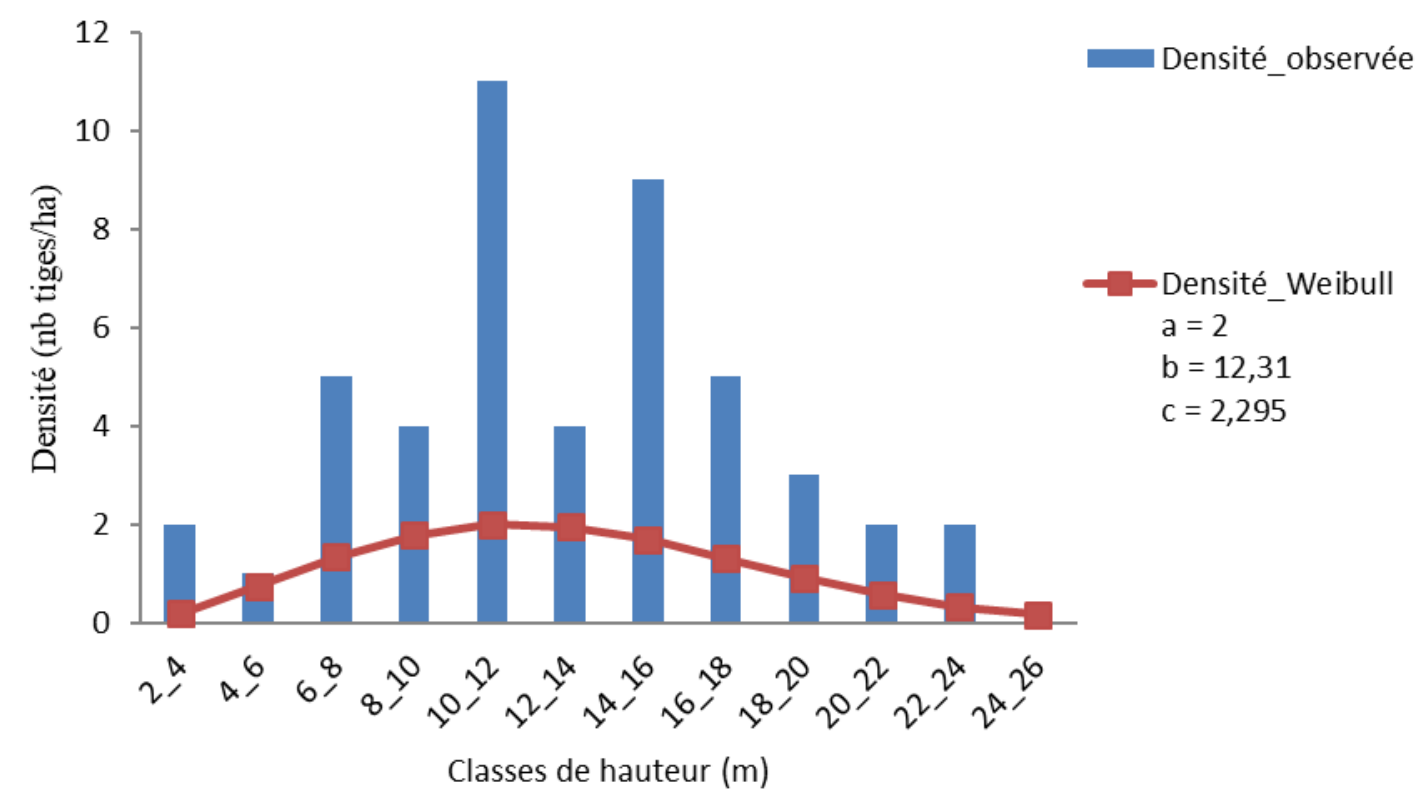

Figure 5 : Classes de hauteur d'Isoberlinia doka

L'analyse de la figure $\mathrm{n}^{\circ} 5$ montre que les individus de la population d'I. doka les plus représentés à l'hectare appartiennent respectivement aux classes de hauteur comprise

\section{DISCUSSION}

Les résultats de l'inventaire floristique montrent que la flore de la végétation de cette zone comprend 51 espèces réparties en 41 genres et 21 familles. En considérant l'abondance des espèces, le peuplement est dominé par les Combretaceae (17,31\%), les Mimosaceae $(15,38 \%)$ et les Ceasalpinoidea (9,61\%). L'abondance des Combretaceae et des Mimosaceae peut être expliquée par le mode de dissémination des espèces appartenant à ces familles. En effet, les Combretaceae sont caractérisées par leurs fruits ailés facilement disséminées par le vent. Les aspects de la dominance des Combretaceae et des Mimosaceae observés peuvent être également dus à leur faculté de régénération naturelle par semis, par drageonnage et/ou par rejets des souches (Bellefontaine, 2005). Les résultats obtenus corroborent ceux de Douma et al., (2010) dans la réserve totale de Tamou (Niger) entre $10-12 \mathrm{~m} ; 8-10 \mathrm{~m}$ et $12-14 \mathrm{~m} ; 14-16 \mathrm{~m}, 6-$ $8 \mathrm{~m}$ et $16-18 \mathrm{~m}$; 4-6 $\mathrm{m}$ et $18-20 \mathrm{~m} ; 20-22 \mathrm{~m}$. les individus de taille supérieure à $22 \mathrm{~m}$ et inférieure à $4 \mathrm{~m}$ sont quasi absents.

et de Inoussa (2011) dans les forêts claires du Parc National du W du Niger qui ont montré que les familles les plus représentées sont les Combretaceae et les Mimosaceae ; cependant, ils diffèrent de ceux obtenus par Djonattane (2012) dans la réserve totale de Tamou (Niger) qui a montré la dominance des Rubiaceae, suivis des Combretaceae et des Cesalpiniaceae. Les auteurs (Guinko ,1984 et Saadou, 1990) ont rattaché la zone du parc au domaine des forêts claires et des savanes que White (1983) place dans le centre d'endémisme régional soudanien. Ceci explique la faible densité des individus d' $A$. africana et d'I. doka, car le parc du $\mathrm{W}$ se situe dans une zone de transition (nordsud). Au sud du Parc W, les surfaces couvertes par I. doka sont relativement étendues White(1983). En effet Fandohan (2006) a trouvé dans la forêt classée de Wari-maro une densité de 142 sujets/ha des individus d'A. 
africana soit plus de $35 \%$ des individus inventoriés à l'hectare. L'indice de diversité de Shannon obtenu de 1,38bit, celui de Simpson de $1-\mathrm{D}=0,93$ et l'équitabilité de 0,81 montrent que la diversité spécifique du milieu est bonne. Ceci a été vérifié en comparant l'indice de Simpson à l'équitabilité de Pielou. Les résultats obtenus rapprochent ceux de Namata (1998) dans le Parc du W du Niger qui a obtenu un indice de Shannon 1,23 bit, soit 70,36\% à l'échelle Shannon et 88,84 à l'échelle de Simpson et sont nettement inférieure à ceux obtenus par Inoussa (2011) dans les forêts claires du Parc National du W du Niger avec des valeurs de l'indice de Shannon variant de 3,6 à 5,5 bits. Les structures en diamètre des deux populations ont été ajustées à la distribution de Weibull et présentent une allure en forme de cloche avec une prédominance des individus de diamètre compris entre $24-36 \mathrm{~cm}$ pour la population d' $A$. africana et $30-36 \mathrm{~cm}$ pour celle d'I. doka. Les individus de classes de diamètre $(24-30 \mathrm{~cm}$ et $30-36 \mathrm{~cm} ; 18-24 \mathrm{~cm}$ et $36-42 \mathrm{~cm} ; 42-48 \mathrm{~cm} ; 48-54 \mathrm{~cm} ; 54-60 \mathrm{~cm})$ observés chez $A$. africana et de diamètre $30-36 \mathrm{~cm}$, $24-30 \mathrm{~cm}, 18-24 \mathrm{~cm}$ et $36-42 \mathrm{~cm} ; 12-18 \mathrm{~cm}$ et $42-48 \mathrm{~cm}$; 48-54 cm ; 54-60 cm observés chez I doka nous permet d'affirmer que la population des deux espèces est à prédominance jeune, ceci est en désaccord avec les résultats de Bonou (2007) dans la forêt classée de Lama au Benin qui a montrer une population vieillissante. Les structures en hauteur des deux peuplements prouvées par nos résultats ont été ajustés à la distribution de Weibull et présentent une dissymétrie gauche caractéristique $(1<\mathrm{c}<3,6)$ des peuplements à prédominance jeunes avec des individus de hauteur moyenne comprise entre 10-14 $\mathrm{m}$ au niveau des deux espèces. Kakpo (2011) a trouvé que les classes de la population d' $A$. africana de hauteur comprise entre 22-24 m sont les plus représentées dans la forêt d'Ithcèdè Tandis que les individus d'I. doka les plus représentés à l'hectare appartiennent aux classes de hauteur comprises entre 16 à $18 \mathrm{~m}$ de hauteur caractéristique de la population vieille. Nos résultats se rapprochent également de ceux des travaux de Glele Kakai et Sinsin (2009) au Benin qui ont montré qu'en savane (même caractéristiques que le Parc w), la classe de hauteur 12-14 m est plus représentée pour l'espèce Isoberlinia doka. Dans l'ensemble notre étude montre une population d' $A$. africana et I. doka jeune caractérisée par des individus de petite taille par rapport à la partie sud du parc w plus arrosée (Kakpo 2011). Par ailleurs (G. Guissou ,2001) a montré que la faible densité des individus de petite hauteur au niveau des deux peuplements est due par le fait que les feuilles sont broutées par les animaux et en particulier les antilopes qui mangent les jeunes pousses et les rejets. Les modes de régénération par drageons sont plus observés dans le parc W chez les deux espèces avec peu de cas de germination par graines chez A. africana. Ceci est en accord avec les résultats de massalatchi qui a montré que d'une part Afzelia africana se régénère quand-même par graines au cas où celles-ci bénéficient de la protection de la coque et mais surtout par drageonnage et d'autre part Isoberlinia doka se régénère dans la plupart des cas par drageonnage. En somme les deux espèces présentent une faible densité de régénération par graines. En la dynamique de cette régénération des espèces est assujettie à des menaces auxquelles elles font face. A travers des observations directes sur le terrain, une fois les graines disséminées, elles sont broutées par les animaux, en particulier chez $I$. doka où les graines sont très sensibles à l'humidité, ce qui fait qu'elles perdent facilement leur faculté de germination par contact avec le minimum d'humidité une fois tombées. Aussi, le passage du feu d'aménagement dans le parc du W détruit les graines et les plantules des deux espèces (Diouf 2013).

\section{CONCLUSION ET PERSPECTIVES}


Cette étude s'inscrit dans le cadre de la recherche d'une meilleure connaissance de la biodiversité du Parc National du W du Niger en général et des espèces Afzelia africana et Isoberlinia doka en particulier pour leur conservation dans les écosystèmes. L'étude a permis de recenser 51 espèces reparties en 41 genres et 21 familles. Les familles les mieux représentées sont les Combretaceae, les Mimosaceae et les Ceasalpinoidea. La végétation présente une bonne diversité spécifique à travers le calcul des indices de diversité et de l'équitabilité.

La densité de pieds d'A. africana est de 45,17 pieds/ha et 17,49 pieds/ha chez I. doka. Quant à l'analyse des structures horizontales et verticales des deux populations, elles sont toutes en forme de cloche et présentent une dissymétrie gauche avec une prédominance des individus jeunes de petites tailles en diamètre tout comme en hauteur. Les individus de grandes tailles donc âgées sont peu représentés. Les taux de régénération naturelle pour les deux espèces sont faibles. De façon générale, toutes les données issues de ces travaux vont constituer une situation de référence sur l'état desdites espèces et leur suivi via la mise en place d'un dispositif permanent. En effet, pour un suivi saisonnier du dynamique inter et intraannuelle de leur habitat, il est opportun d'installer des placeaux permanents. D'ores et déjà les sites des relevés phytosociologiques et dendrométriques installés au cours de cette thèse peuvent être retenus dans ce sens. Au terme de cette étude, des perspectives sont dégagées en vue d'envisager des mesures de conservation des deux espèces. Ainsi une étude de la régénération pourrait être menée afin de déterminer le stade auquel les plantules sont plus vulnérables. Ceci permettra de les pérenniser en faisant de la régénération naturelle assistée dans les zones périphériques du PNWN et dans les zones écologiques du Niger où les deux espèces ont été découvertes. On peut aussi installer des pépinières dans des zones du Niger où les peuplements ont été découverts (Gorou bassounga et Banban Rafia) dans le cadre d'un programme de reboisement avec l'accord de la communauté riveraine suivis d'une enquête ethnobotanique afin de déterminer les différentes exploitations faites de ces deux espèces par les populations.

- Envisager l'émergence des nouvelles dynamiques de production qui garantissent la conservation et l'utilisation durable desdites espèces et d'apporter les éléments indispensables à leur gestion.

- Mettre en exergue le devenir des forêts claires au sein desquelles se trouvent lesdites espèces dans le parc W. En effet, le suivi de la végétation dans les régions semi-arides, s'impose aujourd'hui comme une nécessité pour les décideurs politiques et les acteurs du développement tout comme pour les scientifiques. Ceci a besoin que des investigations périodiques soient réalisées dans les différentes formations forestières à toutes les échelles.

- Impliquées les populations riveraines dans les plans d'actions de toute activité de conservation et de protection du Parc à travers le développement de l'écotourisme dans la zone et la prise en compte de leurs problèmes socioéconomiques

- Renforcer la capacité des agents forestiers

\section{REFERENCES BIBLIOGRAPHIQUES}

Agresti A., 1990: Categorical Data Analysis, John Wiley \& Sons, New York, 13 p.
Arbonnier, M., 2002. Arbres, arbustes et lianes d'Afrique de l'Ouest. 2è édition, CIRADMNHN UICN, 541p. 
Barbier N., 2006. Auto-organisation et interactions spatiales des végétations semi-arides. Thèse de doctorat, Université Libre de Bruxelles ; 111p.

Benoît M., 1998. Statut et usage du sol en périphérie du parc du «W $»$ du Niger. Introduction à la genèse de l'espace « sauvage » dans la région du « $\mathrm{W} » \mathrm{du}$ Niger, Tome 2, ORSTOM, Niamey, 30 p.

Braun-Blanquet J., 1932. Plant sociology. The study of plant communities. Ed. McGray Hill,

Brice Sinsin et R.L.Glele Kakai., 2010. Description d'Isoberlinia spp Caesalpiniaceae. Université Abomey Calavi/Benin. Article 7pages.

Caswell H., 2001: Matrix Population Models: construction analysis and interpretation. 2nd Ed. Sinauer Associates, Massachusetts12 pages.

Couteron P. et Kokou $\mathrm{K}$ et Mahamane A., 1992. Parc National du W (Niger) : typologie et cartographie de la végétation du Parc National et de la réserve de faune de Tamou, éd. $M A B$ UNESCO, 98 pages.

Diouf A., 2006. Etude des interactions biologiques au sein d'une végétation périodique du Niger par l'analyse des processus ponctuels, Université Libre Bruxelles 42p.

Diouf A., 2013.Influence du régime des feux d'aménagement sur la structure ligneuse des savanes nord-soudaniennes dans le Parc du W (Sud-ouest NIGER), Université Libre Bruxelles 225p.

Dourma M., 2006. Multiplication par drageonnage de Isoberlinia doka et Isoberlinia tomentosa au sein des formations arborées du Nord-Togo, Laboratoire de botanique, et écologie végétale Faculté des sciences, université de Lomé. Article de recherche, 9 pages.

Douma S, RABI C, Ali M et Koini M., 2009. Caractéristiques écologiques des populations de quatre ligneux fruitiers des parcs agroforestiers de la zone déclassée de la réserve totale de Tamou (Niger) CNRA/Abidjan/Côte d'Ivoire et laboratoire de biologie Garba Mounkaila, département de biologie, FS/UAM, Niamey-Niger. 14pages.

Fandohan B., 2006. Abondance et structure des peuplements de sept espèces ligneuses médicales dans la forêt classée de WariMaro (Région des Monts Kouffé). Th. Ing.

FAO., 2010: Evaluation des ressources forestières. Document de travail.25pages.

Gounot M. ; 1969. - Méthode d'étude quantitative de la végétation. Paris VIe. $303 \mathrm{p}$.

Guinko S. ; 1984.- Végétation de Haute Volta .Thèse de Doctorat ès. Sci. Nat., Université de Bordeaux III. 318 p.

Guissou, T. G., 2001. La symbiose mycorhizienne à arbuscules chez des espèces ligneuses : diversité des glomales, dépendance mycorhizienne, utilisation des phosphates naturels et tolérance à un stress hydrique. Thèse de doctorat soutenue à l'Université de Ouagadougou, 124 pages.

Houinato M.R.B., 2001.- Phytosociologie, écologie, production et capacité de charge des formations végétales pâturées dans la région des Monts Kouffé (Bénin). Thèse de doctorat, Université Libre de Bruxelles, Faculté des Sciences, Section Inter facultaire d'Agronomie, Laboratoire de Botanique Systématique, 219p.

INOUSSA Maman Maârouhi., 2011. Dynamique des forêts claires dans le Parc National du W du Niger. Thèse de doctorat de l'Université Abdou Moumouni de Niamey/Biologie et Ecologie Végétale, 128pages

Jonathan G. AGBODJOGBE., 2012. Analyse de la Structure des Galeries Forestières de la Réserve Totale de Faune de Tamou (RTFT) en République du 
Niger. Thèse pour l'obtention d'un diplôme de master. Biodiversité végéta le tropicale. SEP. UPMC/IRD/UAM. 59pages.

Mahamane A., 2005. Etudes floristique, phytosociologique et phytogéographique de la végétation du Parc Régional du W du Niger. Thèse de doctorat en Sciences Agronomiques et Ingénierie Biologique. Université Libre de Bruxelles, Faculté des Sciences, Ecole Interfacultaire de Bioingénieurs Laboratoire de Botanique Systématique et de Phytosociologie, 484 pages.

Massalatchi Abdou Moumouni, 2017. Germination et développement des plantules d'Afzelia africana Smith et Isoberlinia doka Craib et Stapf, espèces à faible régénération naturelle. MEMOIRE de Master en « Protection de l'Environnement et Amélioration des Systèmes Agraires Sahéliens ».85 pages

Natta A. K., 2003.- Ecological assessment of riparian forest in Benin. Phytodiversity, New York, London.: 439 p.

Ouédraogo A., 2006. Diagnostic de l'état de dégradation des peuplements de quatre espèces ligneuses en zone soudanienne du Burkina Faso. In Science et changements planétaires / Sécheresse. Volume 17. Numéro 4, 485-91.

Ouedraogo O., 2009. Phytosociologie, dynamique et productivité de la végétation du Parc National d'Arly (sudest du Burkina Faso) Doctorat de l'Université de Onagadongou 140p.

Saadou M., 1990.- La végétation des milieux drainés nigériens à l'est du fleuve Niger. Thèse de doctorat, Université Niamey, Niger, 393p.

Saadou \& Mahamane., 2007. Vers la mise en place d'un dispositif national de surveillance environnemental (DANSE). Rapport technique au ROSELT.300p.

Samaila Namata., 1998. Contribution à l'analyse de composition floristique et de la structure des principaux groupements ligneux de la partie du parc national du «W » du Niger comprise entre les rivières de Moussiemou, la Tapoa et la piste GSK. Mémoire de fin de cycle Faculté d'agronomie. 42pages.

Sunday Berlioz KAKPO., 2011. Caractéristiques structurales et écologiques des forêts de Bonou et d'Itchèdè au sud- est du Bénin. Diplome d'ingénieur agronome spécialiste en aménagement et gestion des ressources naturelles. Université d'AbomeyCalalvi, faculté des sciences agronomiques. 63 pages.

UICN., 2002. Rapport de la Réunion Internationale Africaine préparatoire au Sommet Mondial du Développement Durable, Bureau Régional de pour l'Afrique de l'Ouest. University, 215p

White F., 1983. La végétation de l'Afrique. Mémoire accompagnant la carte de végétation de l'Afrique. L'Organisation des Nations Unies pour l'Education, la Science et la Culture (UNESCO). 